\title{
The Suzaku broadband X-ray spectrum of the dwarf Seyfert galaxy NGC 4395
}

\author{
K. Iwasawa ${ }^{1, \star}$, Y. Tanaka ${ }^{2}$, and L. C. Gallo ${ }^{3}$ \\ 1 INAF-Ossservatorio Astronomico di Bologna, via Ranzani 1, 40127 Bologna, Italy \\ e-mail: kazushi.iwasawa@oabo.inaf.it \\ 2 Max-Planck-Institut für extraterrestrische Physik, Giessenbachstraße 1, 85748 Garching, Germany \\ e-mail: ytanaka@mpe.mpg.de \\ 3 Department of Astronomy and Physics, Saint Mary's University, Halifax, NS B3H 3C3, Canada \\ e-mail: lgallo@ap.smu.ca
}

Received 6 May 2009 / Accepted 21 December 2009

\section{ABSTRACT}

\begin{abstract}
We present a Suzaku observation of the dwarf Seyfert galaxy NGC 4395 with an estimated black hole mass of $\sim 10^{5} M_{\odot}$. Rapid and strong X-ray variability with an rms amplitude of $\sim 60$ per cent is observed in the $0.4-10 \mathrm{keV}$ band with the XIS cameras. The shape of the light curve appears to depend on energies. The hard X-ray emission is detected up to $35 \mathrm{keV}$ with the HXD-PIN detector at a similar flux level as observed with the INTEGRAL IBIS. The X-ray spectrum below $10 \mathrm{keV}$ is strongly absorbed by partially ionized $\left(\xi \sim 35 \mathrm{erg} \mathrm{s} \mathrm{cm}^{-1}\right)$ gas with a mean equivalent hydrogen column density of $\sim 2 \times 10^{22} \mathrm{~cm}^{-2}$, when a simple absorption model is applied. The spectral shape is also strongly variable but not a simple function of the source brightness. The spectral variability appears to be accounted for mainly by continuum slope changes, but variability in the ionized absorber may also play some part. The apparently flat spectral slope of $\Gamma \simeq 1.4$ below $10 \mathrm{keV}$, obtained after correcting for absorption, is marginally inconsistent with the $\Gamma \sim 2$ inferred from the 14-35 keV PIN spectrum. If the true spectral slope had been as steep as that measured in the hard X-ray band, there would have been an extra absorption component, which we are unable to detect. Combined with the INTEGRAL measurements, the hard X-ray emission above $10 \mathrm{keV}$ exceeds the optical emission in terms of luminosity and dominates the broadband energy output, unless a large excess of UV disk emission is yet to be detected in the unobservable band. A weak Fe K line is seen at $6.4 \mathrm{keV}$ with the average equivalent width of $110 \mathrm{eV}$, which does not show significant flux changes over the 3-day observation.
\end{abstract}

Key words. galaxies: active - galaxies: individual: NGC 4395 - X-ray: galaxies

\section{Introduction}

NGC 4395 is a dwarf galaxy located at the distance of $4.0 \mathrm{Mpc}$ (Thim et al. 2004) that is known to host a very low luminosity Seyfert nucleus (Filippenko et al. 1993). The active nucleus is located at the centre of the galaxy, which has no obvious bulge component. Unlike LINER-like, low-luminosity AGN, the optical/UV spectrum of NGC 4395 shows broad Balmer emission and high-excitation lines, reminiscent of genuine Seyfert nuclei but of higher luminosity (e.g., Ho et al. 1997a). Using the relation usually applied to higher luminosity AGN (Kaspi et al. 2000), the bolometric luminosity estimated from the $5100 \AA$ luminosity is $5.4 \times 10^{40} \mathrm{erg} \mathrm{s}^{-1}$ (Peterson et al. 2005), which can be powered by a black hole of a few $100 M_{\odot}$ if it accretes close to the Eddington limit.

There have been indications that the mass of the black hole in NGC 4395 may be $10^{5} M_{\odot}$ or lower, including by X-ray timing analysis (Kraemer et al. 1999; Lira et al. 1999). The mass derived from the reverberation mapping technique is found to be $(3.6 \pm 1.1) \times 10^{5} M_{\odot}($ Peterson et al. 2005). However, the low bulge velocity dispersion $\leq 30 \mathrm{~km} \mathrm{~s}^{-1}$ (Filippenko \& Ho 2003) deviates from the extrapolation of the $M-\sigma$ relation (e.g., Tremaine et al. 2002) when this estimate of the black hole mass is adopted. Some researchers (e.g., McHardy et al. 2006) propose a lower mass, of even an order of magnitude below the value obtained from reverberation mapping.

* Present address: ICREA Research Professor at Institut de Ciéncies del Cosmos, Universitat de Barcelona, Martí Franquès, 1, 08028 Barcelona, Spain.
As pointed out by Peterson et al. (2005), the Eddington ratio $\left(L_{\text {bol }} / L_{\text {Edd }}\right)$ is low $1.3 \times 10^{-3}\left[M_{\mathrm{BH}} /\left(3.6 \times 10^{5} M_{\odot}\right)\right]^{-1}$. Provided that the estimate of the bolometric luminosity derived from the $5100 \AA$ luminosity is correct, even for the lower mass estimate $\left(\sim 10^{4} M_{\odot}\right)$, the accretion rate is the order of $10^{-2}$ of the Eddington limit. In terms of the accretion rate, the X-ray source in NGC 4395 corresponds to the low/hard state of X-ray binaries.

The soft X-ray emission observed with ROSAT (Moran et al. 1999; Lira et al. 1999) is weak largely because of strong absorption (Iwasawa et al. 2000). The absorbing material is partially ionized (warm absorber) and neutral (cold) absorption is usually small (a few times $10^{20} \mathrm{~cm}^{-2}$ in $N_{\mathrm{H}}$ ). However, an occasional increase in the cold absorbing column, perhaps due to a small cloud passing across the line of sight, has been observed in one of the XMM-Newton observations (Dewangan et al. 2009).

The X-ray emission is known to be extremely variable on short timescales. The temporal properties of the X-ray emission of NGC 4395 was studied in detail using a long XMM-Newton observation with a consecutive $90 \mathrm{ks}$ duration (Vaughan et al. 2005). The power spectrum of the flux variation shows a break at $\sim 2 \times 10^{-3} \mathrm{~Hz}$, which is much higher than those for other AGN, suggesting a low black hole mass. The rms amplitude of the soft $\mathrm{X}$-ray flux variability exceeds 100 per cent.

In comparison with other AGN, the X-ray spectrum of NGC 4395 below $10 \mathrm{keV}$ appears to be very hard even after 


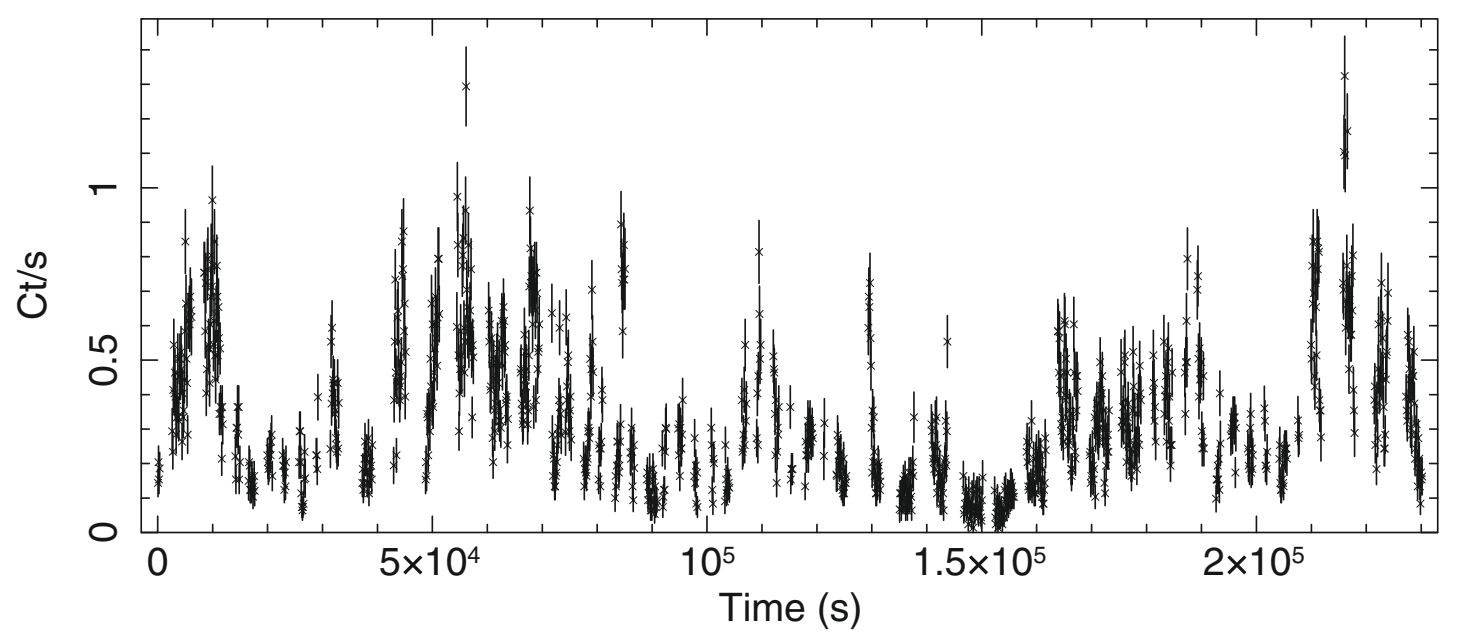

Fig. 1. The X-ray light curve of NGC 4395 during the Suzaku observation. The background-subtracted counts in the $0.4-10 \mathrm{keV}$ band from the three XISs are summed together. Each timebin is of a $100 \mathrm{~s}$ duration.

correcting for absorption, $\Gamma \sim 1.4$ (e.g., Shih et al. 2004; Moran et al. (2005) suggested an even lower value of $\Gamma \sim 0.6)$. Despite the relatively low $2-10 \mathrm{keV}$ flux $\left(\sim 4 \times 10^{-13} \mathrm{erg} \mathrm{s}^{-1} \mathrm{~cm}^{-2}\right)$, NGC 4395 was detected by INTEGRAL IBIS (Beckman et al. 2006; Bird et al. 2007) in 20-40 keV and 40-100 keV bands. This high-energy X-ray detection may imply that the hard spectrum continues into the hard band. The measurement of the spectral shape above $10 \mathrm{keV}$, where it is less affected by absorption, is important to assess the origin of the hard intrinsic spectrum. We observed NGC 4395 with Suzaku to measure the spectral shape above $10 \mathrm{keV}$ and characterise the flux and spectral variability in the softer band by using the X-ray CCD spectrometer over a continuous period of more than $\sim 2.5$ days.

\section{Observation and data reduction}

NGC 4395 was observed with Suzaku (Mitsuda et al. 2006) on 2007 June $02-05$, for a total duration of $230 \mathrm{ks}$. The XIS cameras were operated with the Space-Row Charge Injection in the standard observation mode. The data were taken by the three XISs: two FI CCD cameras (XIS0 and XIS3), one BI CCD camera (XIS1), and the HXD-PIN. The useful exposures obtained from the XIS and the HXD-PIN were $101 \mathrm{ks}$ and $95 \mathrm{ks}$, respectively. NGC 4395 was observed in the "HXD nominal" position on the detector plane, which is optimised for the maximum throughput to the HXD rather than the XIS.

The data from version 2 processing ${ }^{1}$ were used in the analysis. The cleaned event files produced by the data processing with the standard data selection criteria were used. The non X-ray background of the PIN detector was estimated using the "tuned" background model ${ }^{2}$. The background-corrected count rates observed with the three XIS in the $0.4-10 \mathrm{keV}$ band are $0.107 \pm 0.001$ (XIS0), $0.116 \pm 0.001$ (XIS1), $0.109 \pm 0.001$ (XIS3), and $0.031 \pm 0.002$ with the HXD-PIN in the $14-40 \mathrm{keV}$ band.

\section{Results}

\subsection{X-ray variability in the XIS band}

The X-ray source in NGC4395 is strongly variable during the Suzaku observation, as previously observed with other

\footnotetext{
${ }^{1}$ http://www.astro.isas.ac.jp/suzaku/process/caveats

2 http://www.astro.isas.ac.jp/suzaku/analysis/hxd/ pinnxb/tuned/
}

instruments. Since there are many irregular interruptions in data sampling because of the low-Earth satellite orbit, the Suzaku data are not ideal for a detailed timing study of this rapidly variable object on short timescales. We refer to Vaughan et al. (2005) for such a study with the uninterrupted 90-ks XMM-Newton data. Despite the low observation efficiency, the Suzaku data provide continuous coverage of the source for more than twice the XMM-Newton observation.

Figure 1 shows the $0.4-10 \mathrm{keV}$ light curve of NGC 4395 obtained from the three XIS cameras. The resolution is $100 \mathrm{~s}$ and the count rate is the sum of the three XISs with background correction. Large amplitude flux variations are clearly seen. Flux doubling in 100-s and shorter intervals is common (Fig. 2) and consistent with the characteristic timescale of the variability of a few $100 \mathrm{~s}$ (Vaughan et al. 2005).

While the fractional variability throughout the observation is $F_{\text {var }}=0.60 \pm 0.06$ (see Vaughan et al. 2005, for a comparison with the XMM-Newton results), the variability over a shorter duration can be examined for this rapidly variable object. Figure 3 presents the variation in fractional variability amplitude for $5 \mathrm{ks}$ intervals in a time sequence obtained from the light curve in Fig. 1. A few intervals of excess variability correspond to periods of strong, very short-term flaring within 1000-s or shorter duration, notably in the hard X-ray band (see Fig. 4). The typical (e.g., median) source flux during those periods is low. According to the correlation between total rms and flux that is generally found in black hole systems (Uttley \& McHardy 2001), the quantities measured here are on average expected to remain constant. The measured values are clearly variable as expected from the random realisation of a source with a red-noise spectrum in flux variation (e.g., O'Neill et al. 2006). The mean and standard deviation of $F_{\text {var }}$ in Fig. 3 is 0.32 and 0.13, respectively, as indicated in the figure. Nominal fluctuation of $F_{\text {var }}$ could be represented by the standard deviation. All measurements apart from one are within $3 \sigma$ fluctuation. The largest variability recorded in the $80 \mathrm{ks}$ region (Fig. 2) exceeds $3 \sigma$.

The shape of the light curve depends on the chosen energy band. Light curves with $1 \mathrm{ks}$ resolution in the four bands 0.4-1 keV, 1-2.5 keV, 2.5-5 keV, and 5-10 keV are produced and normalized to their respective mean count rates (Fig. 4). The distribution of the source count rate is far more skewed in the soft band than in the hard. This is demonstrated in Fig. 5, in which only the $1-2.5 \mathrm{keV}$ and 5-10 keV light curves are displayed for 


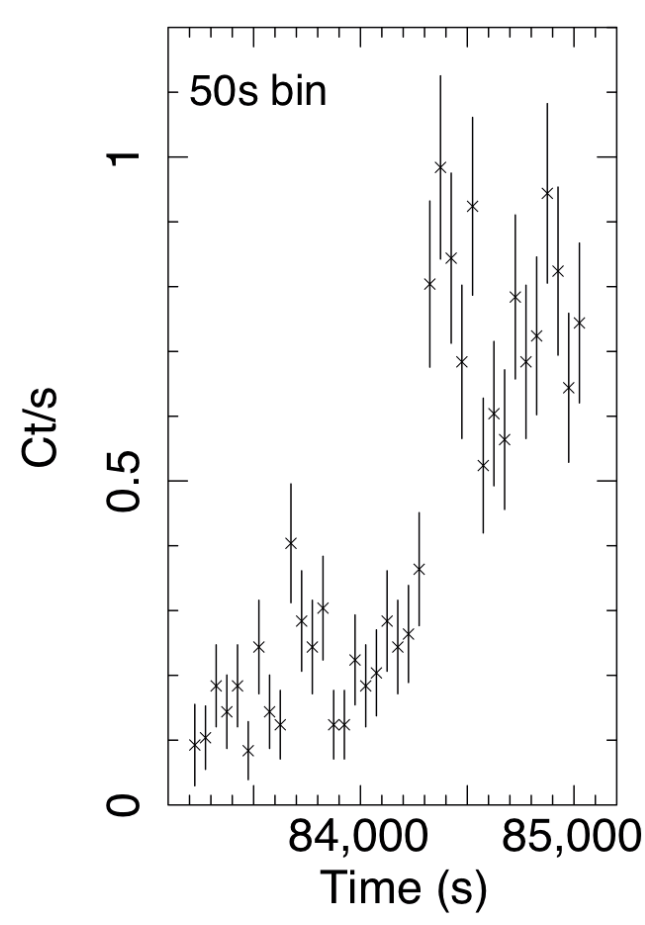

Fig. 2. The blow-up of part of the light curve in Fig. 1 around $84 \mathrm{ks}$ where a rapid and large-amplitude flare occurs. The resolution of the light curve here is $50 \mathrm{~s}$. This strong flare causes the largest $F_{\text {var }}$ in Fig. 3.

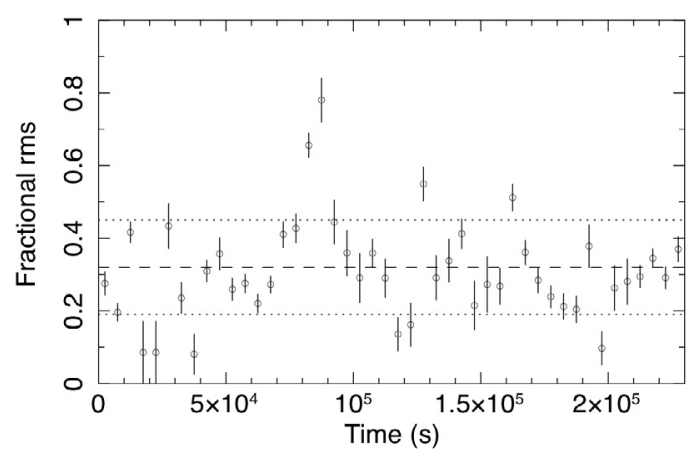

Fig. 3. The variation in the fractional variability amplitude with 5 -ks intervals, obtained from the light curve in Fig. 1. The mean value (0.32) and the standard deviation are marked by the dashed line and dotted lines, respectively.

clarity. The distribution in the $1-2.5 \mathrm{keV}$ curve is well matched to log-normal, while that for the $5-10 \mathrm{keV}$ band is close to being a Gaussian. The (rebinned) correlation diagram on the right also demonstrates that $1-2.5 \mathrm{keV}$ flux is correlated with $5-10 \mathrm{keV}$ flux exponentially rather than linearly, reflecting the difference in light-curve shape mentioned above. The $0.4-1 \mathrm{keV}$ band distribution is even more skewed than at $1-2.5 \mathrm{keV}$, but the low count rate means that distortion by the Poisson noise could be responsible for the extra skewness.

It is known that the light curves of accreting compact objects generally exhibit a log-normal distribution (e.g., Uttley et al. 2005), which is skewed towards the brighter end, as seen in the 1-2.5 keV light curve. We note that where a light curve has a lognormal nature, the asymmetry of the count distribution will be reduced as the fractional variability becomes significantly small (see simulated light curves with the fractional variability of 17 , 41, and 64 per cent in Fig. 4 in Uttley et al. 2005). However, in NGC 4395 , the 5-10 keV band variability remains strong, and

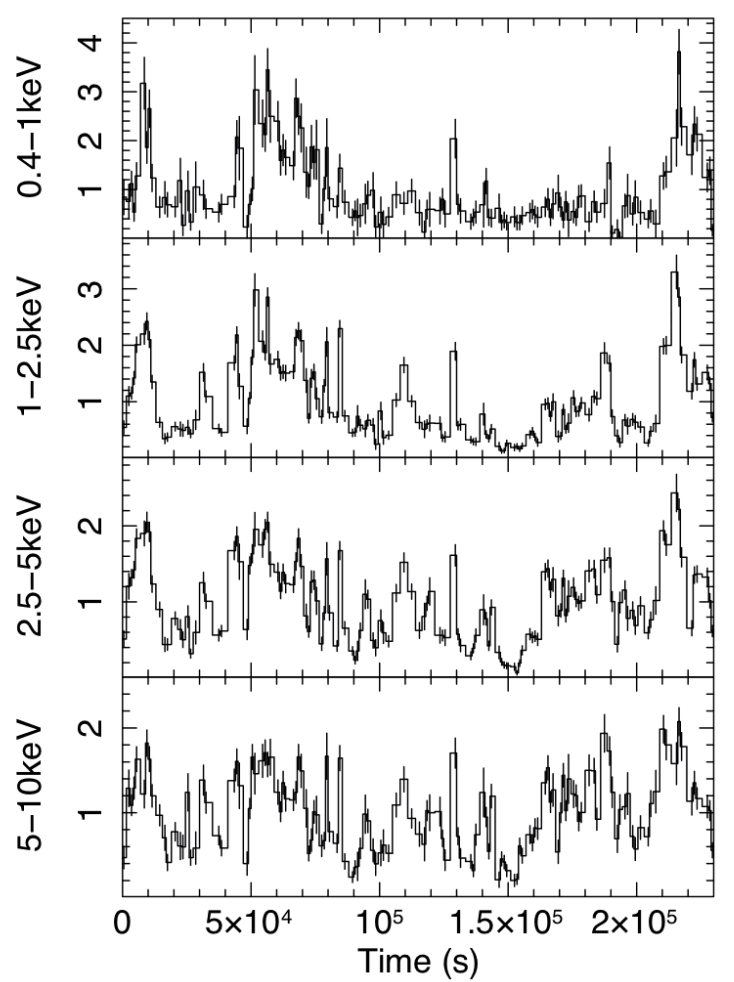

Fig. 4. The $\mathrm{X}$-ray light curves in four energy bands $0.4-1 \mathrm{keV}$, $1-2.5 \mathrm{keV}, 2.5-5 \mathrm{keV}$, and 5-10 keV (from top to bottom) normalized to the mean count rate. The time resolution is $1 \mathrm{ks}$. The mean count rates in the respective bands are $0.019,0.109,0.118$, and 0.079 count s$^{-1}$. The $y$-axis range for each panel is adjusted for visual clarity and varies between the panels. The relative amplitude of the respective light curves can be quantified in Fig. 6 .
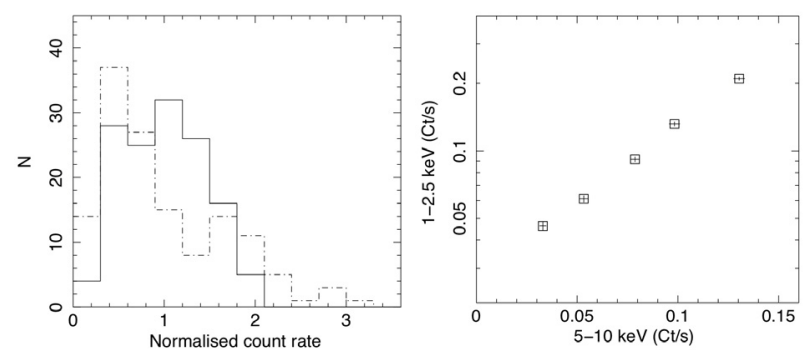

Fig. 5. Left: the distribution of the normalized count rates in the 1-2.5 keV (dash-dotted line) and 5-10 keV (solid line) bands in Fig. 4. It is clear that the distribution in the soft-band light curve is strongly skewed and well matched by a log-normal shape, while that for the hard band is close to being symmetric (i.e., a Gaussian). Right: the correlation diagram between the count rate in the 5-10 keV and $1-2.5 \mathrm{keV}$ band. The data have been incrementally rebinned according to the 5$10 \mathrm{keV}$ count rate, and the five bins contain 27, 27, 27, 27, and 28 measurements in ascending order. Note that the $y$-axis $(1-2.5 \mathrm{keV}$ count rate) is in a logarithmic scale while the $x$-axis $(5-10 \mathrm{keV}$ count rate) is in linear scale.

the reduction in the fractional variability (from 70 per cent in the soft band to 40 per cent in the 5-10 keV, Fig. 6) may not be large enough to account for the change observed. As described below, variability in the ionized absorber could alter the shape of the distribution. Another cautionary note is whether the observation is sufficiently long to cover the timescale on which the stationarity of the time series is achieved, unlike measurements for Galactic binaries. 


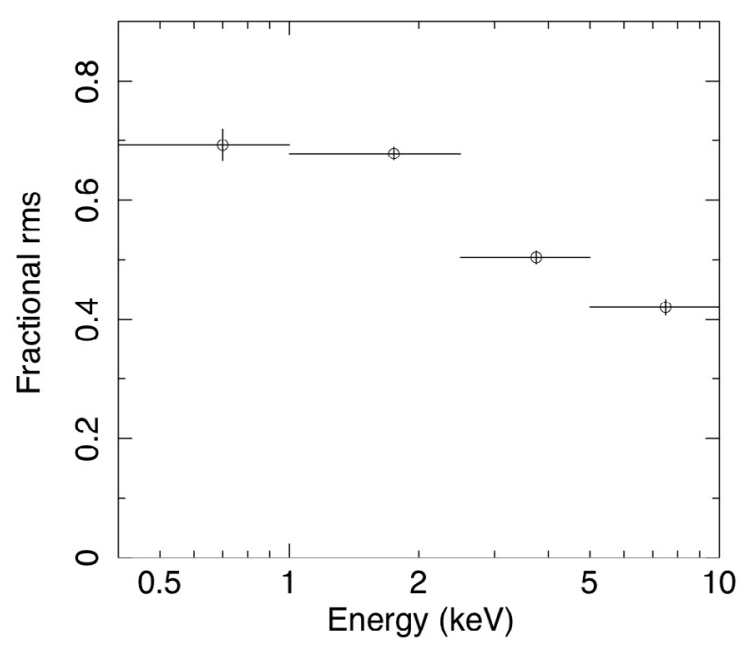

Fig. 6. The fractional rms variability amplitude $\left(F_{\text {var }}\right)$ as a function of energy, obtained from the light curves in $1 \mathrm{ks}$ resolution (see Fig. 4).

The log-normal shape of a distribution is expected in multiplicative products of random events, and, in the context of black hole systems, a model in which fluctuations in the accretion rate are amplified as they propagate inwards has been proposed (e.g., Lyubarskii 1997). In contrast, the symmetric shape of the hardband light curve may be unusual. A superposition of random shots is expected to produce a Gaussian distribution, as seen in the hard X-ray light curve. While the soft and hard X-ray emission in NGC 4395 appear to behave independently on some occasions (Fig. 4), they are statistically correlated (Fig. 5 right). The correlation occurs in a way that the soft X-ray variability is stretched to higher fluxes, i.e., in an exponential manner relative to the hard X-ray (Fig. 5 right). Seyfert galaxies generally shows an approximately linear correlation in two bands with an offset, which is attributed to a constant component such as reflection, while a power-law relation, which is generally expected (Uttley et al. 2005), is more successful in reproducing data for the highly variable NGC 4051 (Taylor et al. 2003).

We note that in Fig. 4, the two relatively broad, intervals containing high flux at 50-90 ks and 160-190 ks have different energy dependences (also see Sect. 3.4). Very short-period/rapid flickering at the 80-90 ks intervals (where the largest variability is recorded in Fig. 3; see also Fig. 2) appears more pronounced at higher energies. The flaring at $90-100 \mathrm{ks}$ is only clear for the two harder bands.

The overall variability amplitude is larger at lower energies, as can be seen in Fig. 6. The fractional variability is similar within the two low energy bands at $\sim 70$ per cent, and declines towards higher energies to $\sim 40$ per cent in the 5-10 keV band.

\subsection{PIN band variability}

The entire observation is divided into 5 time-segments: the first four segments are of $50 \mathrm{ks}$ duration and the last of $30 \mathrm{ks}$. The 13-33 keV count rates from the PIN for these five intervals are plotted in Fig. 7. The error bars in the count rate are statistical only. There appears to be a flux increase by $\sim 0.02 \mathrm{cts}^{-1}$ in the final $80 \mathrm{ks}$ of the observation. In this energy band, 5 per cent of the non X-ray background of the PIN is about $0.018 \mathrm{ct} \mathrm{s}^{-1}$, which is the conservative estimate of the uncertainty given the exposure of the individual data points. The possible flux variation is comparable to this, but the reality of the apparent flux

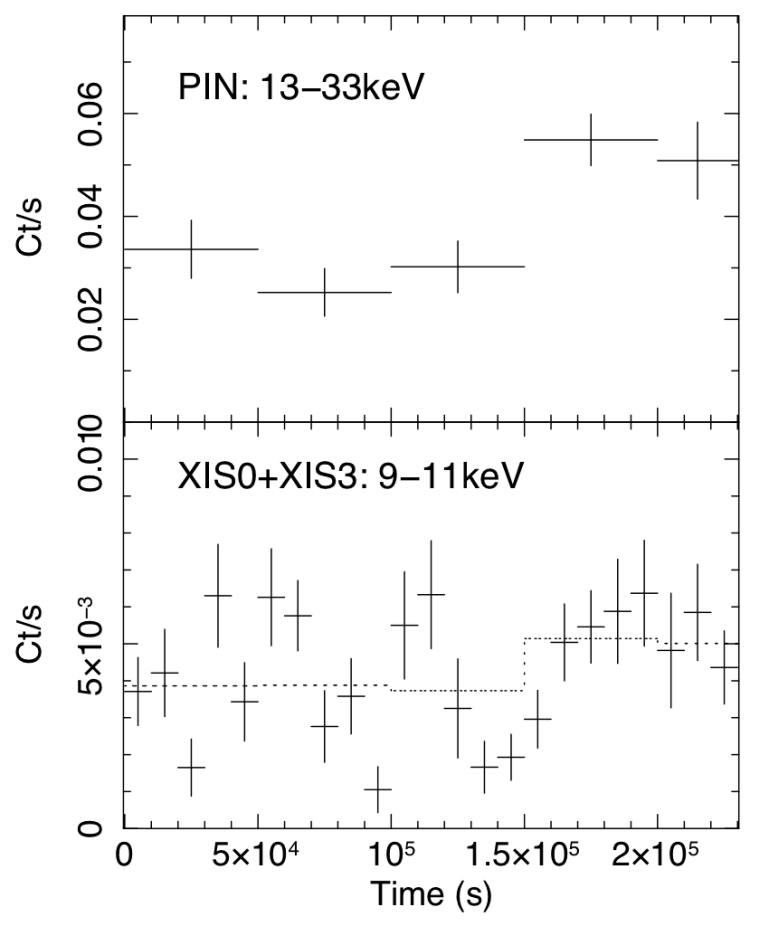

Fig. 7. The light curves of NGC 4395 in the $13-33 \mathrm{keV}$ band from the HXD-PIN (upper panel) and the 9-11 keV band from the XIS0 and the XIS3 (bottom panel). The dotted line indicates the mean counts over the same interval as that of the HXD-PIN light curve.

variation is uncertain. As a comparison, the light curve for the 9-11 keV band, which was obtained by the XIS0 and XIS3 (the signal from the FI-CCD of the XIS1 is very noisy in this band), is also shown in Fig. 7. Flux variability of $\simeq 30 \pm 5$ per cent is still present at the highest energy end of the XIS bandpass when viewed on the $5 \mathrm{ks}$ time resolution. On the $50 \mathrm{ks}$ timescale, the amplitude is lower, but a similar variability pattern between the two light curves indicates that the hard X-ray variability in the PIN band may be real.

\subsection{The time-averaged energy spectrum}

\subsubsection{The XIS data}

A simple power law is unacceptable in describing the full-band data mainly because of the strong absorption at low energies (Fig. 8). The best-fit model photon index $\Gamma \sim 0.7$ indicates that the overall spectrum is unusually hard. Because of the strong absorption and hard spectrum, the spectral analysis here focuses mainly on the two FI CCD cameras, XISO and XIS3.

Introducing a warm absorber and a Fe $\mathrm{K}$ emission-line provides a good description of the $0.4-10 \mathrm{keV}$ band spectrum (Fig. 9). The power-law continuum has a photon-index of $\Gamma=$ $1.40_{-0.04}^{+0.05}$. One of Tim Kallman's XSTAR grid tables (grid25, which is the photoionization grid computed by XSTAR with a power-law ionizing source and the turbulent velocity of the ionized gas of $\left.200 \mathrm{~km} \mathrm{~s}^{-1}\right)^{3}$ is used to model the warm absorber. The absorbing column of the warm absorber is $N_{\mathrm{H}, \mathrm{W}}=$ $2.1_{-0.2}^{+0.3} \times 10^{22} \mathrm{~cm}^{-2}$, and the ionization parameter $\log \xi=1.53_{-0.5}^{+0.6}$ $\left(\mathrm{erg} \mathrm{cm} \mathrm{s}^{-1}\right)$. A small cold absorption of $N_{\mathrm{H}}=5.4 \times 10^{20} \mathrm{~cm}^{-2}$, in addition to the Galactic column $\left(N_{\mathrm{H}}=1.9 \times 10^{20} \mathrm{~cm}^{-2}\right.$, for the

\footnotetext{
3 http://heasarc.gsfc.nasa.gov/docs/software/xstar/ xstar.html
} 


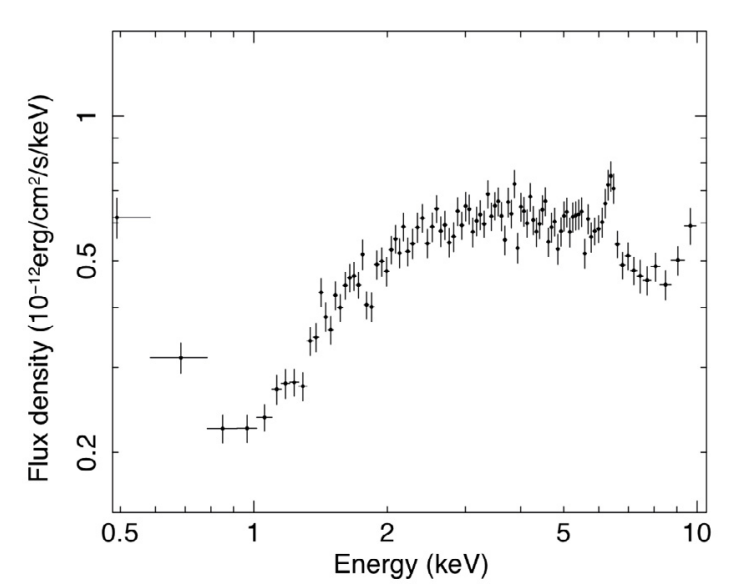

Fig. 8. The $0.5-10 \mathrm{keV}$ spectrum of NGC 4395 observed with the Suzaku XIS. The data are obtained from the XISO and XIS3, and plotted in units of flux density after correcting for the detector response. This correction may have some uncertainty but is accurate enough to show the major spectral features in the spectrum.

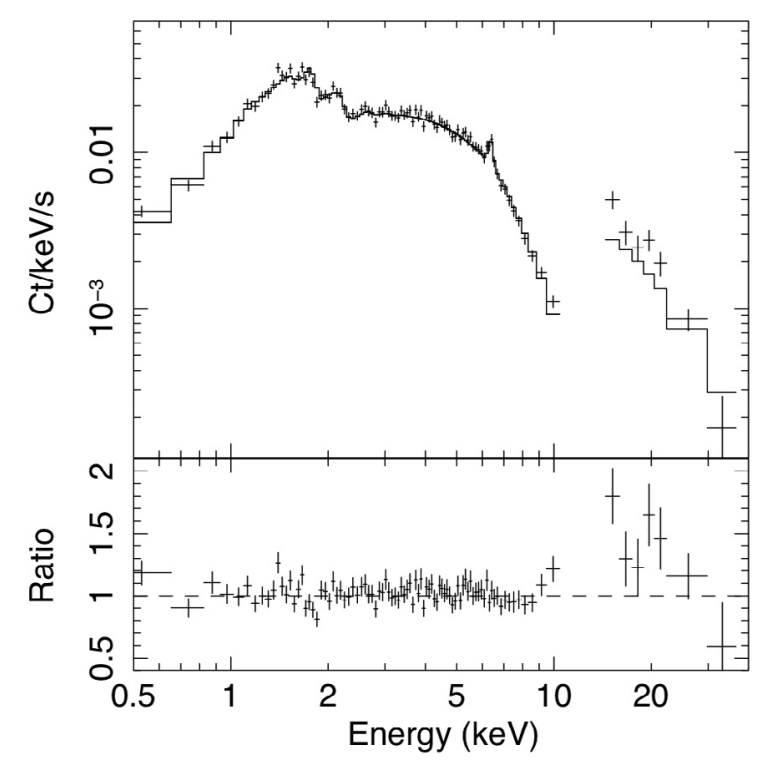

Fig. 9. The $0.5-35 \mathrm{keV}$ spectral data of NGC 4395 obtained with the XIS and HXD-PIN. The solid-line histogram shows the model of a power law with the Fe K line modified by the warm absorber folded through the detector responses, which fits the XIS data. The crosscalibration constant is assumed to be 1:1.18 (XIS:PIN).

LAB map by Kalberla et al. 2005) is also required. The Fe K line, modelled with a Gaussian profile, has a centroid energy at $6.36 \pm 0.04 \mathrm{keV}$, a line width $\sigma=0.09 \pm 0.05 \mathrm{keV}$ (or $F W H M \sim$ $10000 \mathrm{~km} \mathrm{~s}^{-1}$ ), and line intensity $6.4_{-1.8}^{+2.2} \times 10^{-6} \mathrm{ph} \mathrm{s}^{-1} \mathrm{~cm}^{-2}$, which corresponds to an equivalent width of $E W=115_{-32}^{+40} \mathrm{eV}$. The quality of fit ( $\chi^{2}=406.0$ for 407 degrees of freedom) is good (Table 1). We note that there is a hint of systematic hardening above $9 \mathrm{keV}$ (see Fig. 8 and the residual plot in Fig. 9). However, the data in this range have small statistical weight in the full-band spectrum and the deviation does not have an impact on the quality of the overall fit.

The observed flux is $5.1 \times 10^{-13} \mathrm{erg} \mathrm{s}^{-1} \mathrm{~cm}^{-2}$ in the $0.5-2 \mathrm{keV}$ band and $4.4 \times 10^{-12} \mathrm{erg} \mathrm{s}^{-1} \mathrm{~cm}^{-2}$ in the $2-10 \mathrm{keV}$ band. The $2-10 \mathrm{keV}$ flux is similar to that during the long ASCA observation $\left(4.7 \times 10^{-12} \mathrm{erg} \mathrm{s}^{-1} \mathrm{~cm}^{-2}\right.$, Shih et al. 2002) and slightly ( $\sim 20$ per cent) lower than that during the long XMMNewton observation $\left(5.6 \times 10^{-12} \mathrm{erg} \mathrm{s}^{-1} \mathrm{~cm}^{-2}\right.$, Vaughan et al. 2005).

\subsubsection{PIN data}

The 15-35 keV spectrum measured with the HXD-PIN appears to be much steeper than that below $10 \mathrm{keV}$, albeit with a large uncertainty. Fitting a power law provides a reasonable fit to the data with a photon index of $\Gamma=2.2 \pm 0.5\left(\chi^{2}=14.5\right.$ for the 8 degrees of freedom). The inclusion of any spectral curvature or break does not improve the fit. The $20-40 \mathrm{keV}$ flux, estimated by the simple power-law model, is $5.9 \times 10^{-12} \mathrm{erg} \mathrm{s}^{-1} \mathrm{~cm}^{-2}$, which is similar to those measured previously by the INTEGRAL IBIS (Beckman et al. 2006; Bird et al. 2007).

The broad-band spectrum is examined by combining the XIS and the PIN spectrum. Since the observation efficiency of the PIN is slightly lower than that of the XIS, good time intervals for the PIN are also used to produce the XIS spectrum analysed here ( $~ 85$ per cent of the original XIS exposure).

When extrapolating the best-fit model of the XIS spectrum (Fig. 9), the lower end of the PIN data are well above the extrapolation. The cross-calibration constant of $1.18^{4}$ between the PIN and the XIS is adopted, which was measured for the Crab nebula at the HXD nominal pointing position. Provided this cross calibration is reliable, a spectral hump in the $10-25 \mathrm{keV}$ range is required, which may be supported by the possible spectral hardening seen at the highest energy end of the XIS data (Figs. 8 and 9). However, we caution that the uncertainty in the PIN background estimate may significantly influence whether we detect a spectral hump for this faint source. The source fraction of the total PIN counts in the energy band of interest is only $\sim 9$ per cent. \pm 3 per cent uncertainty in the background estimate translates into the cross-calibration constant in the range of $0.8-1.6$ being allowed. A close match between the extrapolated model and the 15-20 keV PIN data can indeed be obtained when the crosscalibration constant is increased to $\sim 1.5$.

\subsection{Spectral variations}

As inferred by the study of light curves in various energy bands, the spectral shape of the X-ray source in NGC 4395 is also variable. Furthermore, the spectral variability does not appear to correlate with the source brightness (see Sect. 3.1).

The throughput of the Suzaku XIS is insufficient to track the spectral changes on the time-scale of the most rapid flux variability (of the order of $100 \mathrm{~s}$ ). Instead, the spectral variability was investigated on a timescale of 5-ks using X-ray colours. The four energy bands $0.4-1 \mathrm{keV}, 1-2.5 \mathrm{keV}, 2.5-5 \mathrm{keV}$, and 5$10 \mathrm{keV}$ were used in the $\mathrm{X}$-ray colour analysis. The X-ray colour is defined to be $\mathrm{HR}=(\mathrm{H}-\mathrm{S}) /(\mathrm{H}+\mathrm{S})$, where $\mathrm{H}$ and $\mathrm{S}$ are the background-corrected count rates collected from the three XIS cameras in two bands of choice and $\mathrm{H}$ is always of the higher energy. Four HR (HR1, HR2, HR3, and HR4) are derived as shown in Table 2.

The X-ray colour measurements are plotted in Fig. 10, along with the full-band light curve. Four correlation-diagrams between the X-ray colours and the source brightness are presented in Fig. 11. Overall, there is no clear correlation between the

\footnotetext{
4 ftp://legacy.gsfc.nasa.gov/suzaku/doc/xrt/ suzakumemo-2008-06.pdf
} 
Table 1. Spectral fit to the Suzaku XIS time-averaged spectrum.

\begin{tabular}{ccccccccc}
\hline \hline Data & $\Gamma$ & $\begin{array}{c}N_{\mathrm{H}} \\
\mathrm{cm}^{-2}\end{array}$ & $\begin{array}{c}N_{\mathrm{H}, \mathrm{W}} \\
\mathrm{cm}^{-2}\end{array}$ & $\begin{array}{c}\xi \\
\mathrm{erg} \mathrm{s}^{-1} \mathrm{~cm}\end{array}$ & $\begin{array}{c}E_{\mathrm{FeK}} \\
\mathrm{keV}\end{array}$ & $\begin{array}{c}\sigma \\
\mathrm{keV}\end{array}$ & $\begin{array}{c}I_{\mathrm{FeK}} \\
\mathrm{ph} \mathrm{s}^{-1} \mathrm{~cm}^{-2}\end{array}$ & $\chi^{2} /$ d.o.f. \\
\hline Total & $1.40_{-0.04}^{+0.05}$ & $5.4_{-3.0}^{+2.8} \times 10^{20}$ & $2.1_{-0.2}^{+0.3} \times 10^{22}$ & $34_{-4}^{+5}$ & $6.36_{-0.04}^{+0.04}$ & $0.09_{-0.05}^{+0.05}$ & $6.4_{-1.8}^{+2.2} \times 10^{-6}$ & $406.0 / 407$ \\
\hline
\end{tabular}

Notes. The spectrum was obtained by combining the XIS0 and XIS3 data. The model consists of a power law with a Gaussian for the Fe K $\alpha$ line, modified by warm and cold absorption. The cold absorption column is denoted by $N_{\mathrm{H}}$, which is in excess of the Galactic column of $1.85 \times 10^{20} \mathrm{~cm}^{-2}$. Tim Kallman's XSTAR grid tables ("grid 25") with the turbulent velocity of $200 \mathrm{~km} \mathrm{~s}^{-1}$ is adopted for the warm absorber modelling.

Table 2. The definition of the four X-ray colours.

\begin{tabular}{cccc}
\hline \hline HR & $\begin{array}{c}\mathrm{S} \\
\mathrm{keV}\end{array}$ & $\begin{array}{c}\mathrm{H} \\
\mathrm{keV}\end{array}$ & $\chi^{2} /$ d.o.f. \\
\hline HR1 & $0.4-1$ & $1-2.5$ & $42.2 / 45$ \\
$\mathrm{HR} 2$ & $1-2.5$ & $2.5-5$ & $323 / 45$ \\
$\mathrm{HR} 3$ & $2.5-5$ & $5-10$ & $115 / 45$ \\
HR4 & $0.4-1$ & $5-10$ & $117 / 45$ \\
\hline
\end{tabular}

Notes. Each colour indicator $(\mathrm{HR})$ is defined by $(\mathrm{H}-\mathrm{S}) /(\mathrm{H}+\mathrm{S})$, where $\mathrm{H}$ and $\mathrm{S}$ are count rates measured in the two energy bands given above. $\chi^{2}$ values for a constant hypothesis are also included.

Table 3. The XIS 1-10 keV spectra of the six time-intervals.

\begin{tabular}{lcccc}
\hline \hline Interval & $\Gamma$ & $\log \xi^{a}$ & $N_{\mathrm{W}}{ }^{b}$ & $F_{2-10}{ }^{c}$ \\
\hline 1 & $1.50 \pm 0.07$ & $1.6 \pm 0.1$ & $1.6 \pm 0.2$ & 4.8 \\
2 & $1.67 \pm 0.06$ & $1.7 \pm 0.1$ & $1.2 \pm 0.2$ & 5.2 \\
3 & $1.32 \pm 0.08$ & $1.3 \pm 0.1$ & $2.0 \pm 0.2$ & 3.3 \\
4 & $1.08 \pm 0.06$ & $1.0 \pm 0.1$ & $2.4 \pm 0.2$ & 2.6 \\
5 & $1.16 \pm 0.06$ & $1.0 \pm 0.1$ & $2.4 \pm 0.2$ & 4.9 \\
6 & $1.58 \pm 0.06$ & $1.6 \pm 0.1$ & $1.3 \pm 0.2$ & 5.6 \\
$\Gamma$ & - & $1.47 \pm 0.04$ & $1.40 \pm 0.04$ & \\
$\log \xi^{a}$ & $1.5 \pm 0.1$ & - & $1.2 \pm 0.1$ & \\
$N_{\mathrm{W}}{ }^{b}$ & $2.2 \pm 0.3$ & $2.2 \pm 0.1$ & - & \\
$\chi^{2} /$ d.o.f. & $671.8 / 676$ & $707.2 / 676$ & $698.1 / 676$ & \\
\hline
\end{tabular}

Notes. Results of fitting a single warm absorber model to the $1-10 \mathrm{keV}$ XIS spectra from the six time-intervals are presented. One of the three parameters, $\Gamma, \xi$, and $N_{\mathrm{W}}$, is a free parameter when fitting the six spectra, besides the normalizations of the power-law continuum. The other two are tied together between the spectra and reported below. Observed 2-10 keV flux is also given. ${ }^{(a)}$ In unit of $\mathrm{erg} \mathrm{s}^{-1} \mathrm{~cm} .{ }^{(b)}$ In unit of $10^{22} \mathrm{~cm}^{-2}$. (c) The 2-10 keV flux in unit of $10^{-12} \mathrm{erg} \mathrm{cm}^{-2} \mathrm{~s}^{-1}$.

spectral variability and source flux, although some systematic trends can be found:

1) HR values increase towards lower flux down to the 0.4 $10 \mathrm{keV}$ count rate of $\sim 0.2 \mathrm{ct} \mathrm{s}^{-1}$, except for HR1, 2) below this flux, HR drops, except for HR3. The former trend is consistent with spectral pivoting, i.e., the spectral slope change as a function of source brightness. However, the turnover of the HR value in the low flux range suggests further complication to a simple spectral pivoting. Below $1 \mathrm{keV}$, there is an emission component from photoionized gas, detected through XMM-Newton RGS measurement of OVII (Bianchi \& Guaunazzi 2007), which is detached from the variable nuclear emission and expected to be stable. This could partly explain the HR turnover, namely in HR1 and HR4, as the stable component becomes visible when the nuclear flux is low.

In the time sequence, there is considerable flaring in the 40-80 ks interval, where the spectrum remains soft, as shown by generally low values of HR (Fig. 10). However, the softest

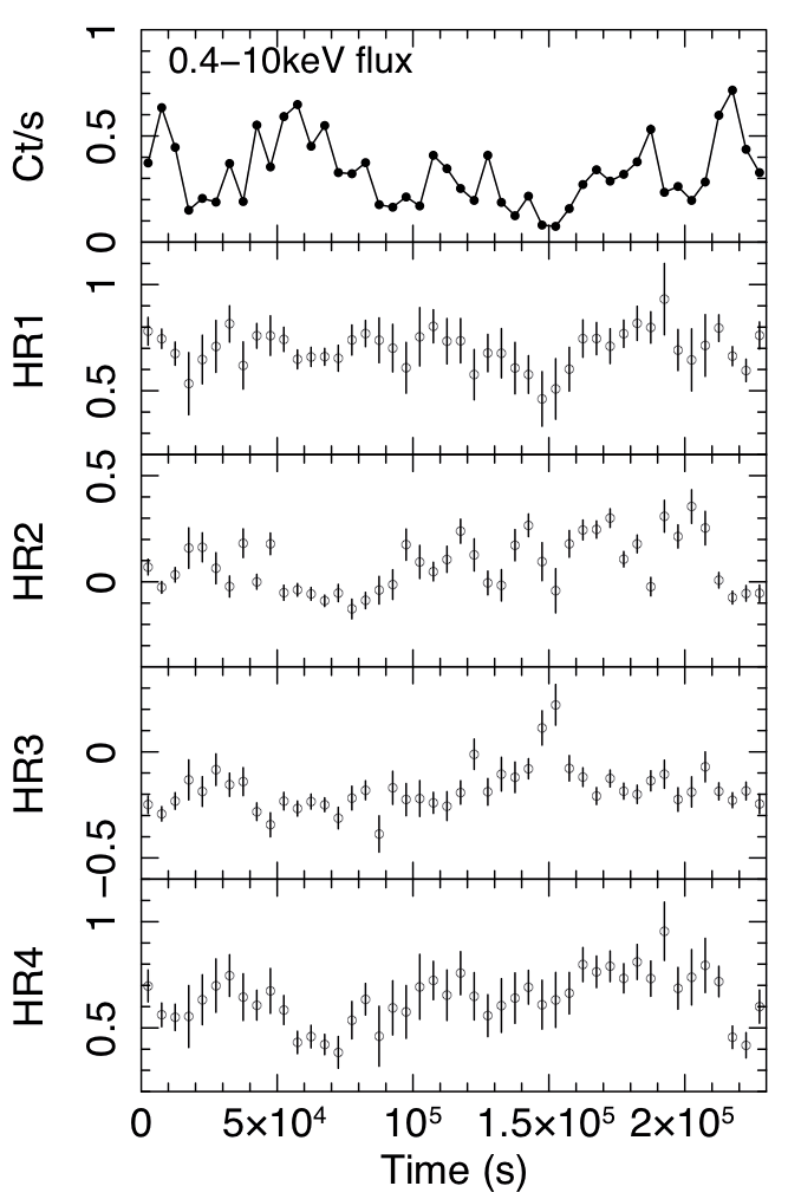

Fig. 10. The temporal variations in the $0.4-10 \mathrm{keV}$ count rate and the four X-ray colours (HR1, HR2, HR3, and HR4) at 5 ks resolution (from top to bottom).

period, indicated by the HR curves, seems to last for a while ( $20 \mathrm{ks})$ after the flare peak. Similar HR behaviour may also occur during the decay of the large flare at the end of the observation. These are seen in HR2, which shows the most significant variation (see Table 2), and possibly in HR4. The colour HR2 is taken from the energy range where the source count rate is the highest, which is probably the main reason for the large significance, but where also the warm absorber modifies the spectrum the most. In the simplest picture of photoionization, the ionization parameter should change in response to variations in the illuminating source and the ionic column of the absorber should also change on the recombination timescale. The complicated HR behaviour may thus also originate from the variability in absorption.

In an attempt to identify the main driver of the spectral variability in NGC 4395, we divided the observation into six intervals and examined their spectra, by applying the simple model 

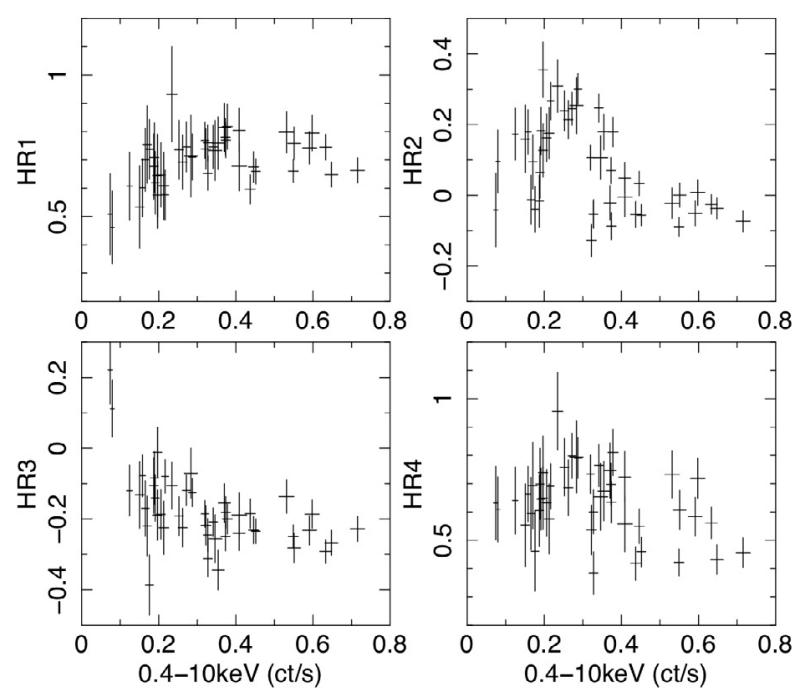

Fig. 11. The three X-ray colour indicators HR1, HR2, HR3, and HR4 (see Table 2) plotted against the $0.4-10 \mathrm{keV}$ count rate.

Table 4. The XIS spectra from the second and fifth intervals.

\begin{tabular}{lcccc}
\hline \hline Interval & $\Gamma$ & $\log \xi^{a}$ & $N_{\mathrm{W}}^{b}$ & \\
2 & $1.75 \pm 0.07$ & $1.6 \pm 0.5$ & $1.1 \pm 0.2$ & \\
5 & $1.23 \pm 0.07$ & $1.0 \pm 0.1$ & $2.4 \pm 0.3$ & \\
$\Gamma$ & - & $1.56 \pm 0.09$ & $1.47 \pm 0.09$ & \\
$\log \xi^{a}$ & $1.4 \pm 0.3$ & - & $1.1 \pm 0.2$ & \\
$N_{\mathrm{W}}^{b}$ & $2.2 \pm 0.5$ & $2.3 \pm 0.3$ & - & \\
$\chi^{2} /$ d.o.f. & $281.8 / 309$ & $293.2 / 309$ & $283.4 / 309$ & \\
& & & & \\
Interval & $\Gamma$ & $\log \xi^{a}$ & $N_{\mathrm{W}}{ }^{b}$ & $\chi^{2} /$ d.o.f. \\
2 & $1.65 \pm 0.08$ & $1.5 \pm 0.2$ & $2.2 \pm 0.4$ & $275.0 / 308$ \\
5 & $1.34 \pm 0.10$ & $1.0 \pm 0.2$ & as above & \\
2 & $1.66 \pm 0.09$ & $1.3 \pm 0.2$ & $1.8 \pm 0.2$ & $275.5 / 308$ \\
5 & $1.34 \pm 0.09$ & as above & $2.3 \pm 0.3$ & \\
\hline
\end{tabular}

Notes. Results of joint model fitting to the XIS spectra from the second and fifth intervals are shown. For the spectral fitting, the procedure is the same as that described in Table 3. The lower part shows the results when the spectral slope and the warm absorber parameters $\left(N_{\mathrm{W}}, \log \xi\right)$ are left to vary, but either of the two warm absorber parameters is tied between the two spectra. ${ }^{(a)}$ In unit of erg s${ }^{-1} \mathrm{~cm} .{ }^{(b)}$ In unit of $10^{22} \mathrm{~cm}^{-2}$.

which was used to model the time-averaged spectrum (Table 1). All the time intervals are of a $40 \mathrm{ks}$ duration each, except for the last interval, which has a $30 \mathrm{ks}$ duration. The second and fifth intervals roughly correspond to the periods, where the spectrum is persistently soft and hard, respectively, according to HR2.

The six spectra were then fitted jointly by restricting the choice of the model free parameters to the power-law normalisations and one parameter of choice among photon index $\Gamma$, the ionization parameter $\xi$, and the absorption column density $N_{\mathrm{W}}$ of the warm absorber, either of which could be the driver of the spectral variability. To avoid possible contamination by photoionized gas at the low flux intervals, the data below $1 \mathrm{keV}$ were excluded. The cold absorption was fixed to the best-fit model value for the time-averaged spectrum (see Table 1), and all other parameters are tied together between the six spectra. Results are summarised in Table 3. The highest quality of fit is obtained when $\Gamma$ is a free parameter, suggesting that the slope change might be the primary driver of the spectral variability.
However, as discussed for the HR analysis, the slope change may not be the unique cause of the spectral variability. We then took the second and fifth intervals, which have almost identical $5-10 \mathrm{keV}$ flux, $\simeq 2.9 \times 10^{-12} \mathrm{erg} \mathrm{s}^{-1} \mathrm{~cm}^{-2}$ (see Fig. 4) but significantly different values in HR2 (see Fig. 10). The same exercise performed for these two intervals infers that a change in the continuum slope and the absorption depth are comparably good solutions (see Table 4). When either $\xi$ or $N_{\mathrm{W}}$ were allowed to vary between a pair of intervals, in addition to $\Gamma$, the quality of the fit improved by a similar degree. The reduction in $\chi^{2}$ relative to the fit, where only $\Gamma$ is variable between the two spectra (Table 4), is $\Delta \chi^{2} \approx-6$, for which a $F$-test infers 99 per cent confidence in the one additional term. No additional improvement in the fit was attainable when both $\xi$ and $N_{\mathrm{W}}$ were allowed to vary. This may indicate that changes in the warm absorber, at least in one of the two parameters, could be a secondary source of the spectral variability. Which parameter varies of either $\xi$ or $N_{\mathrm{W}}$ in the warm absorber is difficult to determine, because their features are degraded at the CCD resolution. The higher value of $\xi$ for the spectrum with higher flux is qualitatively consistent with the photoionization of the absorbing medium.

Finally, we comment on the spectrum at the minimum of the light curve at $\sim 150 \mathrm{ks}$. During this flux minimum, HR1 shows spectral softening, while HR3 shows strong hardening. The softening could be explained by the increased relative contribution of the stable, photoionized-gas emission to the soft band. On the other hand, the larger value of HR3 is caused by rapid flaring, which only occurs in the $5-10 \mathrm{keV}$ band (see Fig. 4). Naturally, this hard X-ray flare has an extremely flat spectrum $(\Gamma=0.25 \pm 0.15)$, which makes it difficult to explain using the absorption model. In this brief period, the variable continuum does not switch off completely, as significant variability is still present (Fig. 2). Therefore, explanation of the hardening in HR3, invoking a constant component (in this case, the cold reflection from distant matter), which would appear mainly in the 5-10 keV band, does not apply.

\section{Iron line variability}

The iron $\mathrm{K}$ line variability was investigated in time and also at different continuum flux levels. Since the line is weak (Table 1), it is not possible to track the response of the line flux to the extremely rapid continuum variation. The XIS spectra from three time intervals $i-1$ of $0-90 \mathrm{ks}, i-2$ of $90-160 \mathrm{ks}$, and $i-3$ of $160-$ $230 \mathrm{ks}$ were extracted and the line flux measured. The intervals, $i-1$ and $i-3$ contain periods of strong flaring, while $i-2$ represents relatively quiet periods.

The spectral data are restricted to the $3-10 \mathrm{keV}$ range and the continuum and the iron line are modelled by an absorbed power law and a Gaussian, respectively. The line flux is not corrected for absorption. The line fluxes in the three intervals agree and show no sign of variability, as shown in Table 5.

Next, the source light curve was sliced into three count-rate ranges and the line flux from each source brightness range was measured. The count-rate slicing was applied to the light curve in Fig. 1 (in the $0.4-10 \mathrm{keV}$ band with the 100-s time resolution) with the count rate ranges of $\mathrm{L}: \leq 0.30 \mathrm{ct} \mathrm{s}^{-1}, \mathrm{M}: 0.30-0.55 \mathrm{ct} \mathrm{s}^{-1}$, and $\mathrm{H}: \geq 0.55 \mathrm{ct} \mathrm{s}^{-1}$. The line flux measurements were performed in the same way as for the time-ordered spectra. The results are presented in Table 5. There is a hint of line flux increase in the data at the highest source brightness, but within the uncertainty, the line flux does not show significant variability over factor of $\sim 3$ change in average flux. At least, a linear correlation between the continuum and line flux can be ruled out. Table 3 also shows 


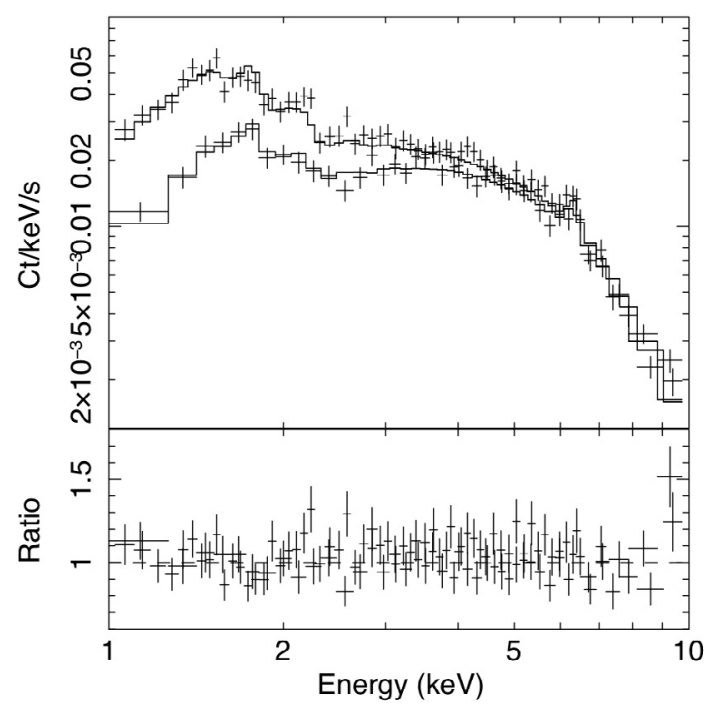

Fig. 12. The count rate spectra for the two intervals (40-80 ks and 160-200 ks), where the 5-10 keV flux is comparable but the HR2 shows largely different values. These two intervals correspond to the second and fifth intervals in Table 3, and investigated in Table 4. The spectra are obtained by combining the data from the XIS0 and XIS3. The solid-line histograms indicate the best-fit model in which the power-law normalization and the absorption column ( $N_{\mathrm{W}}$, see Table 4$)$ are the only independent parameters between the two. The bottom panel shows the residuals in the form of the ratio of the data to the model for the two spectra.

Table 5. Iron K line variability.

\begin{tabular}{lccccc}
\hline \hline Data & $\begin{array}{c}\text { Range } \\
(1)\end{array}$ & $\begin{array}{c}\text { Exposure } \\
(2)\end{array}$ & $\begin{array}{c}\text { Counts } \\
(3)\end{array}$ & $\begin{array}{c}F_{3-10} \\
(4)\end{array}$ & $\begin{array}{c}I_{\mathrm{FeK}} \\
(5)\end{array}$ \\
\hline \multicolumn{2}{l}{ Time order } \\
$i-1$ & $0-90$ & 40.0 & 5243 & 4.0 & $7.5_{-1.9}^{+2.3}$ \\
$i-2$ & $90-160$ & 29.8 & 2450 & 2.5 & $8.1_{-2.2}^{+1.9}$ \\
$i-3$ & $160-230$ & 29.0 & 4117 & 4.3 & $7.1_{-2.1}^{+3.1}$ \\
Flux order & & & & \\
$\mathrm{L}$ & $\leq 0.30$ & 51.8 & 3592 & 2.2 & $5.7_{-1.5}^{+1.7}$ \\
$\mathrm{M}$ & $0.30-0.55$ & 31.7 & 4772 & 4.4 & $7.0_{-2.6}^{+1.8}$ \\
$\mathrm{H}$ & $\geq 0.55$ & 16.2 & 3681 & 6.7 & $9.4_{-3.9}^{+3.4}$ \\
\hline
\end{tabular}

Notes. (1) in unit of $10^{3} \mathrm{~s}$ in the light curve in Fig. 1 for the time intervals, or $\mathrm{ct} \mathrm{s}^{-1}$ for the source count rate range (2) $10^{3} \mathrm{~s}$ (3) backgroundcorrected integrated counts (4) 3-10 keV flux in $10^{-12} \mathrm{erg} \mathrm{s}^{-1} \mathrm{~cm}^{-2}$ (5) Fe $\mathrm{K}$ line flux in $10^{-6} \mathrm{ph} \mathrm{s}^{-1} \mathrm{~cm}^{-2}$. Note: the error in the line flux is of the order of $1 \sigma$.

exposure time, the collected counts, and the flux in the $3-10 \mathrm{keV}$ band.

Ideally, the flux slicing should be performed using the ionizing flux, e.g., the 7-11 keV band flux responsible for ionizing $\mathrm{Fe}$. The much lower count rate in this hard band means however that the time resolution becomes poorer. We made three flux slices based on the 7-11 keV light curve with 10-ks resolution, and investigated the Fe line flux. While the dynamic range of the three flux slices are only a factor of 2 , the line flux remains constant between the flux slices, confirming the result obtained above. The iron $\mathrm{K}$ line is resolved and the inferred $F W H M$ $\left(\sim 10000 \mathrm{~km} \mathrm{~s}^{-1}\right)$ implies a smaller line-emitting radius than the BLR (FWHI $5000 \mathrm{~km} \mathrm{~s}^{-1}$, Ho et al. 1997a). The lack of significant variability in the line flux is unexpected in this compact system. Additional studies of a wider range of ionizing flux variations will be needed to verify the result.

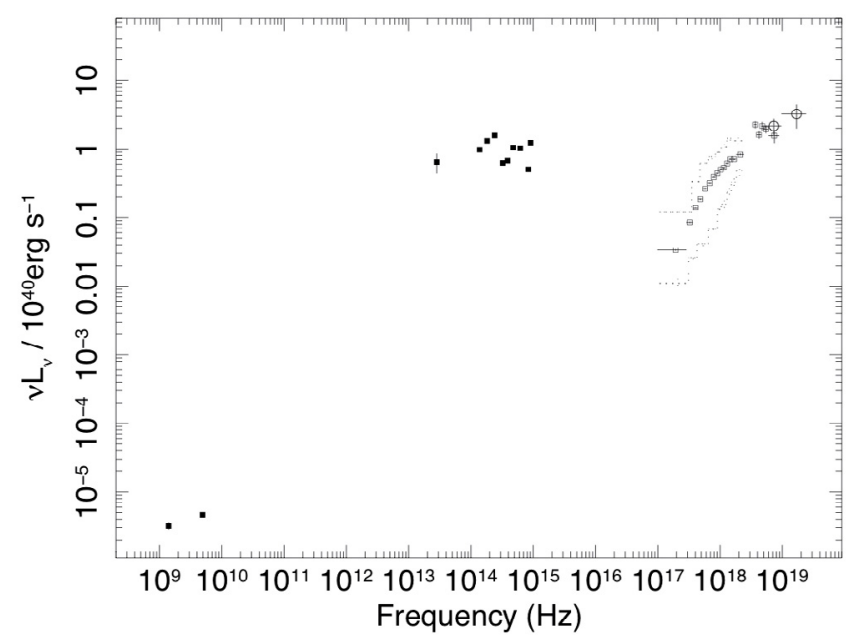

Fig. 13. The energy distribution of NGC 4395 from radio to hard X-rays. The X-ray data (open squares) are measured with Suzaku. The dotted histograms indicate the spectra during the low- and highflux states observed in the Suzaku observation. The $20-40 \mathrm{keV}$ and 40-100 keV data points (open circles) are from INTEGRAL IBIS (Bird et al. 2007). The other points are taken from NED, selecting photometric data from the smallest aperture: SDSS $(u, g, r, i, z)$, HST/ACS (F330W filter, 0.3 arcsec aperture, Muñoz Martin et al. 2007), 2MASS $\left(J, H, K_{\mathrm{S}}, 14 \operatorname{arcsec}\right.$ aperture), MMT (N, 5.3 arcsec aperture, Maiolino et al. 1995), VLBA (5 GHz, Nagar et al. 2005), and FIRST (1.4 GHz, Becker et al. 1995). IRAS fluxes are not included since they are believed to originate in the galaxy, not from the nucleus. The energy distribution of NGC 4395 is unusual for Seyfert galaxies or quasars (e.g., Elvis et al. 1994), the energy output at $10 \mathrm{keV}$ or above being comparable to that in the optical band.

\section{Discussion}

\subsection{Origin of spectral variability}

Strong spectral variability is observed in the XIS band. The general trend is that the spectrum is steeper when the source is brighter, and vice versa, although a close inspection shows that it is not a simple function of the source brightness (Fig. 11 and Table 3). The spectral analysis of the six time intervals (Table 3) appears to show that the spectral variability can be largely accounted for by continuum slope changes. Variability in the warm absorber is less likely, but a good alternative source of the variability and may also change the spectrum. However, for the rapid changes observed in NGC 4395, it is generally difficult to draw strong conclusions using spectra of the CCD spectrometer's resolution as to whether the warm absorber is actually responsible for the variability.

The continuum slope can vary rapidly, as it is probably controlled by the heating/cooling in the hot corona at small radii of the accretion disk. Any change in the physical conditions of the corona can propagate approximately in a light crossing time, which is a few tens of seconds for the radius of 10 gravitational radius in NGC 4395. On the other hand, the warm absorber would need a longer time, the order of the recombination timescale, which depends on the density of the absorbing matter, to respond to the variability of the illuminating source. The $\mathrm{X}$-ray colour HR2, derived from the $1-2.5 \mathrm{keV}$ and $2.5-5 \mathrm{keV}$ data, would be sensitive to any changes in the warm absorber. Possible delays in the response of the hardness ratio to the source flux variation seen in some occasions may be a signature of variations in the warm absrober. 
We now consider that this possible delay of $\simeq 2 \times 10^{4} \mathrm{~s}$ is the response time of the warm absorber. For an ionizing luminosity of $3 \times 10^{40} \mathrm{erg} \mathrm{s}^{-1}$, the calculation of the recombination timescales of the relevant photoionized gas, using the formulae given in Otani et al. (1996) and Krolik \& Kriss (2001), yields the density of $n \sim 10^{7} \mathrm{~cm}^{-3}$ and the distance of the absorbing matter $r \sim 3 \times 10^{-3} \mathrm{pc}$. This density is relatively high but lower than that inferred for the variable warm absorber in NGC 4051 (Krongold et al. 2007) in which the X-ray spectral variability is attributed to variable absorption (but see Ponti et al. 2006).

\subsection{Hard X-ray spectrum and broad-band energy distribution}

The hard X-ray spectrum of NGC 4395 above $10 \mathrm{keV}$, measured with the HXD-PIN, is $\Gamma \sim 2$, the typical value for Seyfert galaxies, while the spectrum below $10 \mathrm{keV}$ is much harder; $\Gamma \simeq 1.4$, even after correcting for the absorption (Table 1). Although the PIN slope is not well constrained, this might be indicative of a spectral break around $10-20 \mathrm{keV}$ or an extra absorption component that we failed to model. A comparison with two INTEGRAL data points in the $20-40 \mathrm{keV}$ and $40-100 \mathrm{keV}$ bands (Fig. 13) suggests that the hard X-ray spectrum may actually continue towards $100 \mathrm{keV}$ with a slope $\Gamma \sim 1.5$, similar to that obtained from the XIS data. However, since the INTEGRAL data were not taken contemporaneously, this is still uncertain due to possible flux variability. A stronger constraint on the hard X-ray spectral shape would therefore be desirable. NGC 4395 is not in the Fermi LAT Bright Source List ${ }^{5}$, which at least rules out such a hard spectrum not extending to $100 \mathrm{MeV}$.

The multiwavelength spectral energy distribution (SED) is constructed by taking photometric data collected from NED (Fig. 13; see also Moran et al. 1999; Lira et al. 1999; Iwasawa et al. 2000). Only the data taken from a small aperture were used to minimize the contamination from the host galaxy, which can be large for this low luminosity nucleus. The IRAS data points are discarded since most of the IRAS fluxes are believed to originate in the galaxy (Lira et al. 1999).

Moran et al. (1999) pointed out the anomalous SED shape of NGC 4395, which differs from that for the luminous QSOs (e.g., Elvis et al. 1994) and also from the low luminosity AGN (Ho 1999), as suggested by the absence of an optical-UV bump and the radio quiet nature of the galaxy (the radio luminosity 5 orders of magnitude below the optical; see also Wrobel \& Ho 2006). Dust reddening, which might suppress the optical light, was discussed by Moran et al. (1999), who did not find compelling evidence of significant reddening. A remarkable feature of the SED in Fig. 13 is that the peak of the energy output occurs at the hard X-ray range, unlike normal Seyfert galaxies and QSOs, in which optical-UV emission dominates the bolometric output. Without soft seed photons from the accretion disk, it would be difficult to explain the production of this strong hard $\mathrm{X}$-ray emission with the thermal Comptonization model (e.g., Haardt \& Maraschi 1991), unless the disk thermal emission escapes our detection into the unobservable shorter wavelength UV range, given the small black hole mass, e.g., the blackbody emission peak would move by a half decade in wavelength, relative to Seyfert galaxies with a black hole mass of $\sim 10^{7} M_{\odot}$.

The distinctive SED shape of NGC 4395 implies that the use of the empirical relation to estimate the bolometric luminosity from the $5100 \AA$ luminosity, which is calibrated by the data for luminous AGN, may be inappropriate. The estimated

\footnotetext{
5 http://fermi.gsfc.nasa.gov/ssc/data/access/lat/ brightsrclist
}

$0.5-100 \mathrm{keV}$ luminosity is $\sim 4 \times 10^{40} \mathrm{erg} \mathrm{s}^{-1}$, which happens to be comparable to the empirical estimate of the bolometric luminosity. If the bulk of the bolometric luminosity indeed comes from the hard X-ray emission, the Eddington ratio remains low, $10^{-3}-10^{-2}$, depending on the $M_{\mathrm{BH}}$ estimate, and lies in an intermediate range between the low accretion-rate (the Eddington ratio of $<10^{-3}$ ) objects such as M 87 and normal Seyfert/QSOs. The radio quietness of the nucleus suggests that the accretion flow is probably not in the form of an advection-dominated type as proposed for objects with a much lower accretion rate. However, we note that NGC 4395 is located on the "fundamental plane of black hole activity" proposed by Merloni et al. (2003). The fundamental plane is parameterised by black hole mass, radio luminosity, and X-ray luminosity. Fitting this relationship suggests that the dim optical emission could be an unusual feature for this active nucleus.

Acknowledgements. We thank all the members of the Suzaku team. We also thank Simon Vaughan, Elisa Costantini and Pierre-Olivier Petrucci for useful discussion and the referee for helpful comments. The data reduction and analysis were performed using the software packages HEASoft provided by NASA's HEASARC. This research made use of the NASA/IPAC Extragalactic Database (NED).

\section{References}

Becker, R. H., White, R. L., \& Helfand, D. J. 1995, ApJ, 450, 559 Beckmann, V., Gehrels, N., Shrader, C. R., \& Soldi, S. 2006, ApJ, 638, 642 Bird, A. J., Malizia, A., Bazzano, A., et al. 2007, ApJS, 170, 175 Elvis, M., Wilkes, B. J., McDowell, J. C., et al. 1994, ApJS, 95, 1 Filippenko, A. V., \& Ho, L. C. 2003, ApJ, 588, L13

Filippenko, A. V., \& Sargent, W. L. W. 1989, ApJ, 342, L11

Filippenko, A. V., Ho, L. C., \& Sargent, W. L. W. 1993, ApJ, 410, L75

Haardt, F., \& Maraschi, L. 1991, ApJ, 380, L51

Ho, L. C. 1999, ApJ, 516, 672

Ho, L. C., Filippenko, A. V., Sargent, W. L. W., \& Peng, C. Y. 1997a, ApJS, 112, 391

Ho, L. C., Filippenko, A. V., \& Sargent, W. L. W. 1997b, ApJS, 112, 315

Iwasawa, K., Fabian, A. C., Almaini, O., et al. 2000, MNRAS, 318, 879

Kalberla, P. M. W., Burton, W. B., Hartmann, D., et al. 2005, A\&A, 440, 775

Kaspi, S., Smith, P. S., Netzer, H., et al. 2000, ApJ, 533, 631

Kraemer, S. B., Ho, L. C., Crenshaw, D. M., Shields, J. C., \& Filippenko, A. V. 1999, ApJ, 520, 564

Krolik, J. H., \& Kriss, G. A. 2001, ApJ, 561, 684

Krongold, Y., Nicastro, F., Elvis, M., et al. 2007, ApJ, 659, 1022

Lira, P., Lawrence, A., O’Brien, P., et al. 1999, MNRAS, 305, 109

Lochner, J. C., Swank, J. H., \& Szymkowiak, A. E. 1989, ApJ, 337, 823

Magdziarz, P., \& Zdziarski, A. A. 1995, MNRAS, 273, 837

Maiolino, R., Ruiz, M., Rieke, G. H., \& Keller, L. D. 1995, ApJ, 446, 561

McHardy, I. M., Koerding, E., Knigge, C., Uttley, P., \& Fender, R. P. 2006, Nature, 444, 730

Merloni, A., Heinz, S., \& di Matteo, T. 2003, MNRAS, 345, 1057

Mitsuda, K., Bautz, M., Inoue, H., et al. 2007, PASJ, 59, 1

Moran, E. C., Filippenko, A. V., Ho, L. C., et al. 1999, PASP, 111, 801

Moran, E. C., Eracleous, M., Leighly, K. M., et al. 2005, AJ, 129, 2108

Muñoz Marín, V. M., González Delgado, R. M., Schmitt, H. R., et al. 2007, AJ, 134,648

Nagar, N. M., Falcke, H., \& Wilson, A. S. 2005, A\&A, 435, 521

Otani, C., Kii, T., Reynolds, C. S., et al. 1996, PASJ, 48, 211

Peterson, B. M., Bentz, M. C., Desroches, L.-B., et al. 2005, ApJ, 632, 799

Ponti, G., Miniutti, G., Cappi, M., et al. 2006, MNRAS, 368, 903

Shih, D. C., Iwasawa, K., \& Fabian, A. C. 2002, MNRAS, 333, 687

Shih, D. C., Iwasawa, K., \& Fabian, A. C. 2003, MNRAS, 341, 973

Taylor, R. D., Uttley, P., \& McHardy, I. M. 2003, MNRAS, 342, L31

Thim, F., Hoessel, J. G., Saha, A., et al. 2004, AJ, 127, 2322

Tremaine, S., Gebhardt, K., Bender, R., et al. 2002, ApJ, 574, 740

Uttley, P., \& McHardy, I. M. 2001, MNRAS, 323, L26

Uttley, P., McHardy, I. M., \& Vaughan, S. 2005, MNRAS, 359, 345

Vaughan, S., Iwasawa, K., Fabian, A. C., \& Hayashida, K. 2005, MNRAS, 356, 524

Wrobel, J. M., \& Ho, L. C. 2006, ApJ, 646, L95 